\title{
Relativistic Treatment of Massive Klein-Gordon Particle by Modified Generalized Hulthen Potential
}

\author{
Akaninyene D. Antia*, Imeh E. Essien and Joseph G. Atat \\ Department of Physics, University of Uyo, Nigeria.
}

Received: October 21, 2016 / Accepted: November 18, 2016 / Published: February 25, 2017.

\begin{abstract}
Approximate bound state solutions of spinless particles with a special case of equal scalar and vector modified generalized Hulthen potential has been obtained under the massive Klein-Gordon equation. The energy eigenvalues and the corresponding wave functions expressed in terms of a Jacobi polynomial are also obtained using the parametric generalization of the Nikiforov-Uvarov (NU) method. Under limiting cases our result are in agreement with the existing literature. Our results could be used to study the interactions and binding energies of the central potential for diatomic molecules in the relativistic framework which have many applications in physics and some others related disciplines.
\end{abstract}

Keywords: Massive Klein-Gordon equation, modified generalized Hulthen potential, eigenvalues, wave function, Nikiforov-Uvarov method and potential barrier.

\section{Introduction}

The studies of exact solutions of the quantum mechanical wave equations such as Schrödinger, Klein-Gordon, Dirac, Salpeter etc. have great importance in atomic and molecular physics and have attracted much attention and interest since the early development of quantum mechanics till today [1-13]. In nuclear and high energy physics, one of the interesting problems is to obtain exact solution of the Klein-Gordon and Dirac equations. When a particle is in a strong potential field, the relativistic effect must be considered, which gives the correction for non relativistic quantum mechanics [14]. In solving non relativistic or relativistic wave equation whether for central or non central potential, various methods are used such as Asymptotic iteration method (AIM) [15], Super symmetric quantum mechanics (SUSYQM) [16] shifted $\frac{1}{N}$ expansion [17], factorization [18], Nikiforov-Uvarov (NU)

\footnotetext{
*To whom correspondence should be addressed: E-mail: antiacauchy@yahoo.com, akaninyeneantia@uniuyo.edu.ng
}

method [19] etc.

In the relativistic quantum mechanics, one can apply the Klein-Gordon equation to the treatment of a zero-spin particle. Recently, many studies have been carried out to explore the relativistic energy eigenvalues and corresponding wave functions of the Klein-Gordon and Dirac equations [14, 20, 21]. The aim of this paper is to obtain the energy eigenvlaues and the corresponding eigen functions for the massive Klein-Gordon particle under modified generalized Hulthen potential in a case of equal scalar and vector using the parametric generalization of the Nikiforov-Uvarov (NU) method.

\section{Brief Review of Nikiforov-Uvarov (NU) Method}

The conventional NU method was presented by Nikiforov and Uvarov [19] and has been employed to solve second order differential equations such as the Schrödinger, Klein-Gordon and Dirac equations etc. The parametric generalization of the NU method is given by the generalized hyper-geometric type equation as [27] 


$$
\begin{gathered}
\Psi^{\prime \prime}(s)+\frac{\left(c_{1}-c_{2} s\right)}{s\left(1-c_{3} s\right)} \Psi^{\prime}(s)+ \\
\frac{1}{s^{2}\left(1-c_{3} s\right)^{2}}\left[-\xi_{1} s^{2}+\xi_{2} s-\xi_{3}\right] \Psi(s)=0
\end{gathered}
$$

According to the NU method, the energy eigenvalues equation and eigen functions, respectively, satisfy the following sets of equations

$$
\begin{gathered}
c_{2} n-(2 n+1) c_{5}+(2 n+1)\left(\sqrt{c_{9}}+c_{3} \sqrt{c_{8}}\right)+ \\
n(n-1) c_{3}+c_{7}+2 c_{3} c_{8}+2 \sqrt{c_{8} c_{9}}=0, \\
\psi(s)= \\
N_{n l} s^{c_{12}}\left(1-c_{3} s\right)^{-c_{12}-\left(c_{13} / c_{3}\right)} P_{n}^{\left(c_{10}-1, \frac{c_{11}}{c_{3}}-c_{10}-1\right)}\left(1-2 c_{3} s\right),
\end{gathered}
$$

where

$$
\begin{gathered}
c_{4}=\frac{1}{2}\left(1-c_{1}\right), c_{5}=\frac{1}{2}\left(c_{2}-2 c_{3}\right), \\
c_{6}=c_{5}^{2}+\xi_{1}, c_{7}=2 c_{4} c_{5}-\xi_{2}, c_{8}=c_{4}^{2}+\xi_{3}, \\
c_{9}=c_{3} c_{7}+c_{3}^{2} c_{8}+c_{6}, c_{10}=c_{1}+2 c_{4}+2 \sqrt{c_{8}}, \\
c_{11}=c_{2}-2 c_{5}+2\left(\sqrt{c_{9}}+c_{3} \sqrt{c_{8}}\right), c_{12}=c_{4}+\sqrt{c_{8}}, \\
c_{13}=c_{5}-\left(\sqrt{c_{9}}+c_{3} \sqrt{c_{8}}\right)
\end{gathered}
$$

and $P_{n}$ is the orthogonal Jacobi Polynomial.

\section{Approximate Solutions of Modified Generalized Hulthen Potential}

The modified generalized Hulthen potential (MGHP) proposed in this work is defined as

$$
V(r)=-V_{0}+V_{1}\left(\frac{a+b e^{-\alpha r}}{g+d e^{-\alpha r}}\right),
$$

where $V_{0}, V_{1}$ are the strength of the potential, $a, b, d, g$ are adjustable potential parameters and $\alpha$ is the screening parameter, the Hulthen potential is a short-range potential in physics which behaves like a coulomb potential for a small values of $r$ and decreases exponentially for a larger values of $r$ [22, 23]. This potential is very important in atom and molecular fields etc. [24-26].

The radial Klein-Gordon equation for a special case of equal scalar and vector potential is given as [28]

$$
\left[E^{2}-M^{2}(r)+2(E+M(r)) V(r)-\frac{\lambda}{r^{2}}\right] R(r)=0,
$$

where $M$ is the mass, $E$ is the relativistic energy, $V(r)$ is the potential under investigation and $\lambda=l(l+1)$, which is the separation constant. In this paper, we assume that the mass of the Klein-Gordon particle depends on the spatial coordinate as [29]

$$
\begin{gathered}
M(r)=M_{0}+M_{1} V(r)= \\
M_{0}+M_{1}\left[-V_{0}+V_{1}\left(\frac{a+b e^{-\alpha r}}{g+d e^{-\alpha r}}\right)\right]
\end{gathered}
$$

The most extensive use of such kind of mass is in the physics of semiconductor quantum well structures [30]. If one set $M_{0}=0, a=0, V_{0}=0, V_{1}=b=g=1$ and mapping $d \rightarrow b$, then our proposed mass is in agreement with the position dependent mass of Ref. [31]. The behavior of the mass function of Eq.(7) with position $r$ is presented in Fig.1.

To obtain the eigenvalues and corresponding eigen functions for this system, we substitute Eqs. (5) and (7) into Eq. (6) to have:

$$
\left[\begin{array}{l}
E^{2}-\left(M_{0}+M_{1}\left[-V_{0}+V_{1}\left(\frac{a+b e^{-\alpha r}}{g+d e^{-\alpha r}}\right)\right]\right)+ \\
2\left(E+M_{0}+M_{1}\left[-V_{0}+V_{1}\left(\frac{a+b e^{-\alpha r}}{g+d e^{-\alpha r}}\right)\right]\right) \\
\left(-V_{0}+V_{1}\left(\frac{a+b e^{-\alpha r}}{g+d e^{-\alpha r}}\right)\right)-\frac{l(l+1)}{r^{2}}
\end{array}\right] R(r)=0 .
$$

Equation (8) has no exact solution for $l \neq 0$ due to the potential barrier, but can be solved approximately by using a suitable approximation scheme. Here we make use of an approximation scheme to deal with the potential barrier as [32]

$$
\frac{1}{r^{2}} \approx \alpha^{2}\left(\frac{g}{g+d e^{-\alpha r}}\right)^{2},
$$




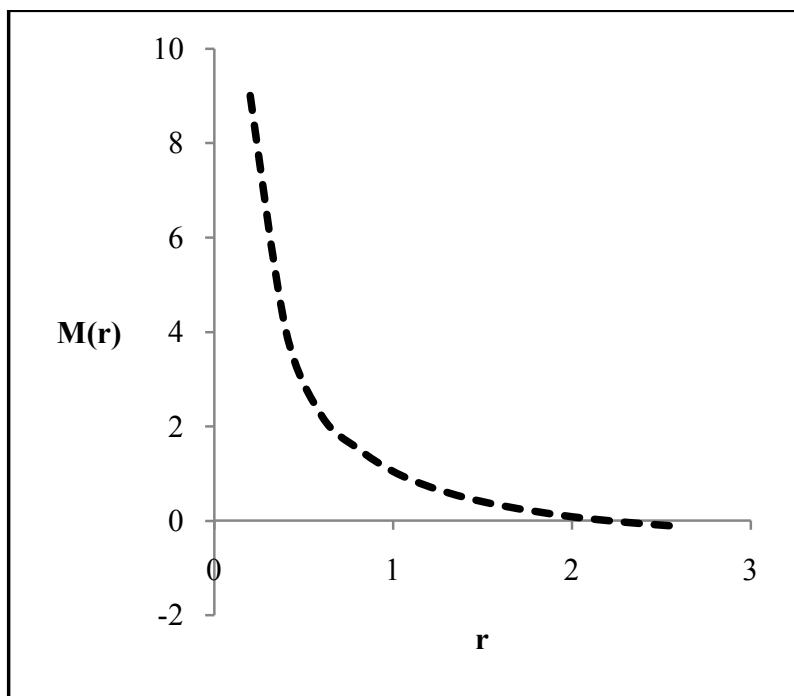

Fig. 1 Variation of mass function $M(r)$ with $r$.

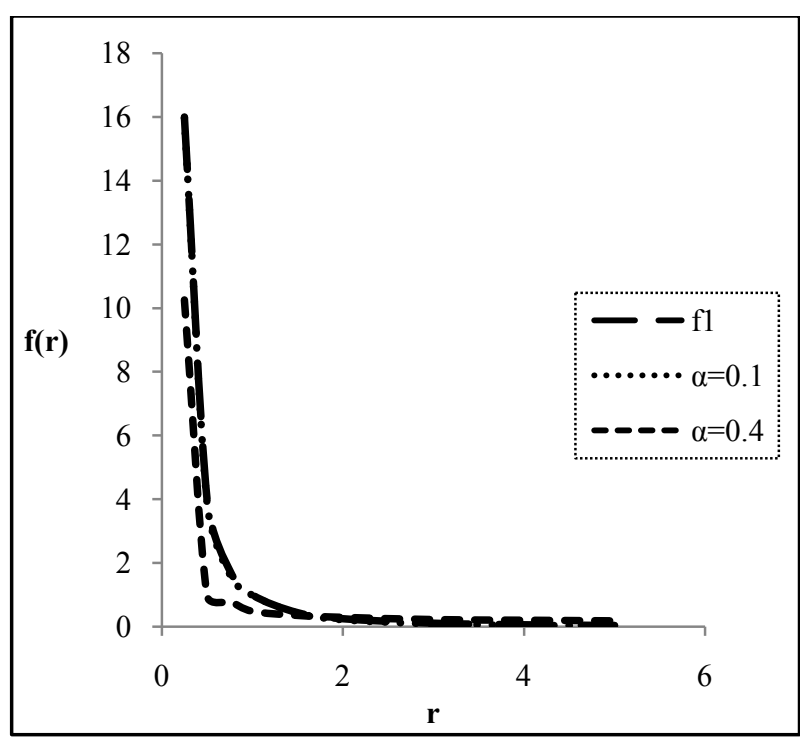

Fig. 2 Comparison of the potential barrier $\mathrm{f} 1=\frac{1}{r^{2}}$ with the approximation for $\alpha=0.1$ and 0.4

where $g=-d$. The comparison of the approximation scheme of Eq. (9) with the centrifugal term ( potential barrier) for various values of $\alpha$ is discussed in Fig. 2. From the graph it is obvious that the approximation is suitable for short range potentials.

Substituting Eq. (9) into Eq. (8) and using the transformation $s=e^{-\alpha r}$ with a simple algebra we obtain the following equation:

$$
\begin{gathered}
\frac{d^{2} R(s)}{d s^{2}}+\frac{\left(1+\frac{d}{g} s\right)}{s\left(1+\frac{d}{g} s\right)} \frac{d R}{d S}+ \\
\frac{1}{s^{2}\left(1+\frac{d}{g} s\right)^{2}}\left[-Q_{1} s^{2}+Q_{2} s-Q_{3}\right] R(s)=0,
\end{gathered}
$$

where

$$
\begin{aligned}
& -\varepsilon^{2}=\frac{E_{n l}^{2}-M_{0}^{2}}{\alpha^{2}} \\
& Q_{1}=\frac{d^{2}}{g^{2}} \varepsilon^{2}+\frac{2\left(E+M_{0}\right)}{\alpha^{2}}\left(\frac{d^{2}}{g^{2}} V_{0}-\frac{d}{c^{2}} V_{1} b\right) \\
& +\frac{d^{2}}{g^{2}} \frac{M_{1} V_{0}}{\alpha^{2}}\left(M_{1} V_{0}-2 M_{0}-V_{0}\right) \\
& +\frac{d}{g^{2}} \frac{2 M_{1} V_{1} b}{\alpha^{2}}\left(M_{0}-M_{1} V_{0}+2 V_{0}\right) \\
& -\frac{M_{1} V_{1}^{2}}{\alpha^{2}}\left(2-M_{1}\right) b^{2} \\
& Q_{2}=-\frac{2 d}{g} \varepsilon^{2}+\frac{2\left(E+M_{0}\right)}{\alpha^{2}}\left(\frac{d}{g^{2}} V_{1} a-\frac{2 V_{0} d}{g}+\frac{V_{1} b}{g}\right) \\
& +\frac{2 d}{g} \frac{M_{1} V_{0}}{\alpha^{2}}\left(2 M_{0}-M_{1} V_{0}+2 V_{0}\right) \\
& +\frac{d}{g^{2}} \frac{2 M_{1}}{\alpha^{2}}\left(M_{1} V_{0} V_{1}-M_{0}-2 V_{0} V_{1}\right) a \\
& +\frac{2}{g} \frac{M_{1} V_{1}}{\alpha^{2}}\left(M_{1} V_{0}-M_{0}-2 V_{0}\right) b \\
& +\frac{2 M_{1} V_{1}^{2}}{\alpha^{2}}\left(2-M_{1} a\right) b \\
& Q_{3}=\varepsilon^{2}+\frac{2\left(E+M_{0}\right)}{\alpha^{2}}\left(V_{0}-\frac{V_{1}}{g} a\right) \\
& +\frac{2}{g} \frac{M_{1}}{\alpha^{2}}\left(M_{0}-M_{1} V_{0} V_{1}-2 V_{0} V_{1}\right) a \\
& +\frac{M_{1} V_{1}^{2}}{\alpha^{2}}\left(M_{1}-2\right) a^{2}- \\
& \frac{M_{1} V_{0}}{\alpha^{2}}\left(2 V_{0}-2 M_{0}-M_{1} V_{0}\right)+l(l+1)
\end{aligned}
$$

Comparing Eq. (10) with Eq. (2) and making use of 
Eq. (5) the following parameters

$$
\begin{aligned}
& c_{1}=1, c_{2}=c_{3}=-\frac{d}{g}, \\
& \xi_{1}=Q_{1}, \xi_{2}=Q_{2}, \xi_{3}=Q_{3}, c_{4}=0, c_{5}=\frac{d}{2 g}, \\
& c_{6}=\frac{d^{2}}{4 g^{2}}+\frac{d^{2} \varepsilon^{2}}{g^{2}}+\frac{2\left(E+M_{0}\right)}{\alpha^{2}}\left(\frac{d^{2}}{g^{2}} V_{0}-\frac{d}{g^{2}} V_{1} b\right)+\frac{d^{2}}{g^{2}} \frac{M_{1} V_{0}}{\alpha^{2}}\left(M_{1} V_{0}-2 M_{0}-2 V_{0}\right) \\
& +\frac{d}{g^{2}} \frac{2 M_{1} V_{1}}{\alpha^{2}}\left(M_{0}-M_{1} V_{0}+2 V_{0}\right) b-\frac{M_{1} V_{1}^{2}}{\alpha^{2}}\left(2-M_{1}\right) b^{2} \\
& c_{7}=\frac{2 d}{g} \varepsilon^{2}-\frac{2\left(E+M_{0}\right)}{\alpha^{2}}\left(\frac{d}{g^{2}} V_{1} a-\frac{2 V_{0} d}{g}+\frac{V_{1} b}{g}\right)-\frac{2 d}{g} \frac{M_{1} V_{0}}{\alpha^{2}}\left(2 M_{0}-M_{1} V_{0}+2 V_{0}\right) \\
& -\frac{d}{g^{2}} \frac{2 M_{1}}{\alpha^{2}}\left(M_{1} V_{0} V_{1}-M_{0}-2 V_{0} V_{1}\right) a-\frac{2}{g} \frac{M_{1} V_{1}}{\alpha^{2}}\left(M_{1} V_{0}-M_{0}-2 V_{0}\right) b-\frac{2 M_{1} V_{1}^{2}}{\alpha^{2}}\left(2-M_{1} a\right) b \\
& c_{8}=\varepsilon^{2}-2 \frac{\left(E+M_{0}\right)}{\alpha^{2}}\left(V_{0}+\frac{V_{1} a}{g}\right)+\frac{2}{g} \frac{M_{1}}{\alpha^{2}}\left(M_{0} M_{1} V_{0} V_{1}+2 V_{0} V_{1}\right) a \\
& +\frac{M_{1} V_{1}^{2}}{\alpha^{2}}\left(M_{1}-2\right) a^{2}-\frac{M_{1} V_{0}}{\alpha^{2}}\left(2 V_{0}+2 M_{0}-M_{1} V_{0}\right)+l(l+1) \\
& c_{9}=-4 \frac{d^{2}}{g^{2}} \frac{\left(E+M_{0}\right)}{\alpha^{2}} v_{0}+\frac{d}{g} 2 \frac{M_{1} V_{1}^{2}}{\alpha^{2}}\left(2-M_{1} a\right) b-\frac{d^{2}}{g^{2}} \frac{M_{1}}{\alpha^{2}}\left(M_{0}-M_{1} V_{0} V_{1}+2 V_{0} V_{1}\right) \frac{a}{g} \\
& +\frac{d^{2}}{g^{2}} \frac{M_{1} V_{1}^{2}}{\alpha^{2}}\left(M_{1}-2\right) a^{2}+\frac{d}{g^{2}} l(l+1)+\frac{d^{2}}{4 g^{2}}-\frac{M_{1} V_{1}^{2}}{\alpha^{2}}\left(2-M_{1}\right) b^{2} \\
& {\left[\varepsilon^{2}-2 \frac{\left(E+M_{0}\right)}{\alpha^{2}}\left(V_{0}+V_{1} \frac{a}{g}\right)+\right.} \\
& c_{10}=1+2\left[\begin{array}{l}
\frac{2}{g} \frac{M_{1}}{\alpha^{2}}\left(M_{0}-M_{1} V_{0} V_{1}+2 V_{0} V_{1}\right) a+\frac{M_{1} V_{1}^{2}}{\alpha^{2}}\left(M_{1}-2\right) a^{2} \\
-\frac{M_{1} V_{0}}{\alpha^{2}}\left(2 V_{0}+2 M_{0}-M_{1} V_{0}\right)+l(l+1)
\end{array}\right] c_{11}=-2 \frac{d}{g}+2\left(\sqrt{c_{9}}-\frac{d}{g} \sqrt{c_{8}}\right) \\
& c_{12}=\left[\begin{array}{l}
\varepsilon^{2}-2 \frac{\left(E+M_{0}\right)}{\alpha^{2}}\left(V_{0}+\frac{V_{1}}{g} a\right)+\frac{2}{g} \frac{M_{1}}{\alpha^{2}}\left(M_{0}-M_{1} V_{0} V_{1}+2 V_{0} V_{1}\right) a+\frac{M_{1} V_{1}^{2}}{\alpha^{2}}\left(M_{1}-2\right) a^{2} \\
-\frac{M_{1} V_{0}}{\alpha^{2}}\left(2 V_{0}+2 M_{0}-M_{1} V_{0}\right)+l(l+1)
\end{array}\right] \\
& c_{13}=\frac{d}{2 g}-\left(\sqrt{c_{9}}-\frac{d}{g} \sqrt{c_{8}}\right)
\end{aligned}
$$


Substituting Eqs. (11) - (15) into Eq. (2), the energy eigenvalues for this system is obtained explicitly as

$$
E_{n l}^{2}-M_{0}^{2}=\alpha^{2} \delta-\frac{\alpha^{2}}{4}\left(\frac{\Lambda+2\left(n+\frac{1}{2}\right) \beta}{\frac{d}{g}\left(n+\frac{1}{2}\right)-\beta}\right)^{2},
$$

where

$$
\begin{aligned}
& \delta=-2\left(\frac{E+M_{0}}{\alpha^{2}}\right)\left(V_{0}+\frac{V_{1} a}{g}\right)+\frac{2}{\alpha^{2} g} M_{1}\left(M_{0}-M_{1} V_{0} V_{1}+2 V_{0} V_{1}\right) a \\
& +\frac{M_{1} V_{1}^{2}}{\alpha^{2}}\left(M_{1}-2\right) a^{2}-\frac{M_{1} V_{0}}{\alpha^{2}}\left(2 V_{0}+2 M_{0}-M_{1} V_{0}\right)+l(l+1) \text {. } \\
& \beta=\left[\begin{array}{c}
-4 \frac{d^{2}}{g^{2}} \frac{\left(E+M_{0}\right)}{\alpha^{2}} v_{0}+\frac{d}{g} 2 \frac{M_{1} V_{1}^{2}}{\alpha^{2}}\left(2-M_{1} a\right) b-\frac{d^{2}}{g^{2}} \frac{M_{1}}{\alpha^{2}}\left(M_{0}-M_{1} V_{0} V_{1}+2 V_{0} V_{1}\right) \frac{a}{g} \\
+\frac{d^{2}}{g^{2}} \frac{M_{1} V_{1}^{2}}{\alpha^{2}}\left(M_{1}-2\right) a^{2}+\frac{d}{g^{2}} l(l+1)+\frac{d^{2}}{4 g^{2}}-\frac{M_{1} V_{1}^{2}}{\alpha^{2}}\left(2-M_{1}\right) b^{2}
\end{array}\right] \\
& \Lambda=-\frac{d}{2 g}-\frac{d}{g} n(n+1)+\frac{2 d}{\alpha^{2} g^{2}}\left(\left(E+M_{0}\right) V_{1}-M_{1} M_{0}-2 M_{1} V_{0} V_{1}+M_{1}^{2} V_{0} V_{1}\right) a \\
& +\frac{2}{\alpha^{2} g}\left(2 M_{1} V_{0} V_{1}-2 g M_{1} V_{1}^{2}-\left(E+M_{0}\right) V_{1}+M_{1} M_{0} V_{1}-M_{1}^{2} V_{0} V_{1}\right) b \\
& +\frac{2 M_{1}^{2} V_{1}^{2}}{\alpha^{2}} a b+\frac{2 d}{\alpha^{2} g} M_{1} V_{1}^{2}\left(2-M_{1}\right) a^{2}+\frac{8 d}{\alpha^{2} g}\left(E+M_{0}\right) V_{0}-\frac{2 d}{g} l(l+1) .
\end{aligned}
$$

And the wave function is obtained using Eqs. (3) and (15) as

$$
\Psi(r)=N_{n l}\left(e^{-\alpha r}\right)^{\mu}\left(1+\frac{d}{g} e^{-\alpha r}\right)^{\frac{1}{2}+v} P_{n}^{(2 \mu, 2 v)}\left(1+2 \frac{d}{g} e^{-\alpha r}\right),
$$

where

$$
\begin{gathered}
\mu=\left[\begin{array}{c}
\varepsilon^{2}-2 \frac{\left(E+M_{0}\right)}{\alpha^{2}}\left(V_{0}+\frac{V_{1} a}{g}\right)+\frac{2}{g} \frac{M_{1}}{\alpha^{2}}\left(M_{0} M_{1} V_{0} V_{1}+2 V_{0} V_{1}\right) a \\
+\frac{M_{1} V_{1}^{2}}{\alpha^{2}}\left(M_{1}-2\right) a^{2}-\frac{M_{1} V_{0}}{\alpha^{2}}\left(2 V_{0}+2 M_{0}-M_{1} V_{0}\right)+l(l+1)
\end{array}\right], \\
v=-\frac{g}{d}\left[\begin{array}{c}
\left.-4 \frac{d^{2}}{g^{2}} \frac{\left(E+M_{0}\right)}{\alpha^{2}} v_{0}+\frac{d}{g} 2 \frac{M_{1} V_{1}^{2}}{\alpha^{2}}\left(2-M_{1} a\right) b-\frac{d^{2}}{g^{2}} \frac{M_{1}}{\alpha^{2}}\left(M_{0}-M_{1} V_{0} V_{1}+2 V_{0} V_{1}\right) \frac{a}{g}\right]^{\frac{1}{2}} \\
+\frac{d^{2}}{g^{2}} \frac{M_{1} V_{1}^{2}}{\alpha^{2}}\left(M_{1}-2\right) a^{2}+\frac{d}{g^{2}} l(l+1)+\frac{d^{2}}{4 g^{2}}-\frac{M_{1} V_{1}^{2}}{\alpha^{2}}\left(2-M_{1}\right) b^{2}
\end{array}\right]
\end{gathered}
$$

and $N_{u}$ is a normalization constant. 


\section{Results and Discussion}

By setting $V_{1}=0$, the potential in Eq. (5) reduces to constant potential [24]. If one set $V_{0}=0, a=g=1, b=d=-1$ and using $\alpha-2 \alpha$ the potential under investigation reduces to Hulthen potential $[10,28]$. Also setting $V_{0}=0, a=b=1$, $g=1, d=-1 \quad$ and mapping $\quad V_{1} \rightarrow-V_{1} \quad$ and $\alpha \rightarrow 2 \alpha$ the potential in Eq.(5) becomes Rosen-Morse potential [33]. Woods-Saxon potential [34] could be deduced from our potential in Eq. (5) if one set $V_{0}=a=0, b=g=d=1$, map $V_{1} \rightarrow-V_{0}$ and mapping $\quad \alpha \rightarrow 2 \alpha$. Similarly, if we set $V_{0}=a=d=0, b=g=1$ and mapping $V_{1} \rightarrow V_{0}$, our potential model reduces to Morse potential [35]. The energy spectrum for these potentials deduced from our potential models could be obtained by using the adjusted parameters in Eqs. (16, 17, 18 and 19).

\section{Conclusion}

In this paper, we have obtained appropriately the bound state solutions of the Klein-Gordon equation under equal and scalar modified generalized Hulthen potential with proper approximation to the centrifugal term (potential barrier) using a powerful NU technique. Explicitly, the energy eigenvalues and the corresponding wave functions expressed in terms of Jacobi polynomial are also obtained. Our approach here offers one of the few examples were the Klein-Gordon equation is solved approximately with position-dependent mass and in an external potential. Finally, in addition to the fundamental importance in physics, the solutions obtained here may play a very vital role in the study of hadrons for both theoretical and experimental physicists.

\section{Reference}

[1] Dirac P A 1958 Principle of Quantum Mechanics (USA: Oxford University Press).

[2] Landau L D and Lifshitz E M 1977 Quantum Mechanics, Non-Relativistic Theory. 3rd Edition (UK: Pergaon).

[3] Greiner W 2000 Relativistic Quantum Mechanics: wave equation (Berlin: Springer).

[4] Yi L Z et al 2004 Phy. Lett. A 333212

[5] Ikot A N 2011 Afri. Rev. Phys. 6221.

[6] Antia A D et al 2015 Adv. Phy. Theor. Appl. 441.

[7] Antia A D et al 2015 Int. J. High Energy Phys. 250.

[8] Ikot A N et al 2013 Few-Body Systems 542041.

[9] Hassanabadi H et al 2011 Commun. Theor. Phys. 55541.

[10] Qiang W C and Dong S H 2009 Phys. Scr. 79045004.

[11] Antia A D et al 2013 Ind. J. Phys. 871133.

[12] Wei G F et al 2008 Phys. Scr. 77035001.

[13] Dong S et al 2007 Phys. Scr. 76393.

[14] Oyewumi K J and Akoshile C O 2010 Eur. Phys. J. A 45 311.

[15] Yasuk F and Bahar M K 2012 Phys. Scr. 85045004.

[16] Hassanabadi H et al 2012 Euro. Phys. J. Plus, 12731.

[17] Mustafa O and Sever R 1991 Phys. Rev. Mod. 444142

[18] Ikhdair S M and Sever R 2008 Int. J. Mod. Phys. 19221

[19] Nikiforov A F and Uvarov V B 1988 Special Functions of Mathematical Physics (Bassel: Birkhauser).

[20] Antia A D et al 2012 Sri Lankan J. Phys. 1327.

[21] Dong S H and Lozaada-Cassou M 2006 Phys. Scr. 74285.

[22] Zarrinkamar S et al 2011 Phys. Scr. 84065008

[23] Ikot A N et al 2015 Acta Physica Polonica A 127674

[24] Flugge S 1974 Practical Quantum Mechanics (Berlin: Springer).

[25] Khanna K M et al 2010 Indian J. Pure and Appl. Phys. 487.

[26] Wamalwa D S and Omolo J A 2010 Indian J. Phys. 85 1513.

[27] Tezcan C and Sever R 2009 Int. J. Theor. Phys. 48337

[28] Ikot A N et al 2011 Elect. J. Theor. 8225.

[29] Kennedy P and Dombey N 2002 J. Phys. A 356645.

[30] Gonul B et al 2002 Mod Phys. Lett. A. 172057.

[31] Meyur S et al 2014 Advances High Energy Physics 952597.

[32] Yazarloo B H et al 2012 Phys. J. Plus 12751.

[33] Debnath S and Biswas B 2012 EJTP 26191.

[34] Berkdemmir A et al 2006 Mod. Phys. Lett. A 212087.

[35] Alhaidari A D 2011 Phys. Lett. B 699309. 